\title{
Brief Solutions to Collatz Problem, Goldbach Conjecture and Twin Primes
}

\author{
Mesut KAVAK*
}

I published some solutions [1] a time ago to Goldbach Conjecture, Collatz Problem and Twin Primes; but I noticed that there were some serious logic voids to explain the problems. After that I made some corrections in my another article [2]; but still there were some mistakes. Even so, I can say it easily that here I brought exact solutions for them out by new methods back to the drawing board.

\section{Goldbach Conjecture}

The main question about Goldbach Conjecture is pretty clear that is each even number sum of two prime numbers?

\subsection{The solution}

Being $p$ is a prime number, for definition of $p_{4}>p_{3} \wedge$ $p_{4}-p_{3}=2 n+2 \wedge n>2 \wedge n \in \mathbb{Z}^{+}$; first number group which is created by $n$ pieces non-prime consecutive positive odd whole numbers, the smallest odd whole number it has is $p_{3}+2$ or $p_{4}-2 n$ and the biggest odd number it has is $p_{3}+2 n$ or $p_{4}-2$, contains greater numbers than last number group which contains $n-1$ pieces consecutive non-prime odd numbers and none of the numbers $n$ group contains, for definition of $p_{2}>$ $p_{1} \wedge p_{2}-p_{1}=2(n-1)+2$ is before $n$ group, and the smallest odd number it has is $p_{1}+2$ or $p_{2}-2(n-1)$ and the biggest one it has is $p_{1}+2(n-1)$ or $p_{2}-2$. Also $n-1$ groups which contain $n-1$ pieces non-prime consecutive odd numbers and have greater numbers than $n$ groups have are possible; but these groups can never exist until $n$ groups emerge because of the above stated reasons and definitions; because $n$ groups also contain 2 pieces $(n-1)_{1}$ and $(n-1)_{2}$ consecutive groups which $n-2$ pieces elements of them are common with $n$ groups.

The difference between $n-1$ and $(n-1)_{m}$ is not to be of prime number there after last number of first group and before first number of last group of emerging two groups; therefore when two groups emerged as $(n-1)_{1}$ and $(n-1)_{2}$, it means that $n$ group already had been emerged spontaneously, and is first $n$ group. Being $(n-1)_{1}$ is the first and $(n-1)_{2}$ is the second group, as last element of $(n-1)_{2}$ group is always bigger than all numbers of $(n-1)_{1}$, it means $(n-1)_{1}$ had already been emerged before $n$ group. Also as $n-1$ group must exist at the place before $(n-1)_{1}$ group, then numbers of $n$ group are always greater than numbers of $n-1$ group for the definition of $n$ and $n-1$ groups. Here all possible $n-k$ groups for $m, k \in \mathbb{Z}^{+}$definition are unique; but $(n-k)_{m}$ groups which have common element with another groups are not unique.

Being $k=0$, for $n-k=n$ groups, 1 piece of selected $n$ pieces consecutive odd numbers from 3 to numbers of $n+1$ group has to be prime number; so if some tables are made like table 1 and table 2 for each $n$, they will help about the main question.

When similar tables for the other $n$ groups are made, and when an addition is done by order between selected $n$ pieces of the smallest consecutive $x$ number group elements and el-
Table 1: The table for $n=2$

\begin{tabular}{l|lllllll}
\hline $\mathbf{x}$ & & & & $\mathbf{y}$ & & & \\
\hline 3 & 3 & 5 & 7 & 9 & 11 & 13 & $\ldots$ \\
5 & 5 & 7 & 9 & 11 & 13 & 15 & $\ldots$ \\
\hline & $\mathbf{x}$ & + & $\mathbf{y}$ & $=$ & $\mathbf{b}$ & & \\
\hline & 6 & 8 & $\mathbf{1 0}$ & $\mathbf{1 2}$ & 14 & $\ldots$ & $2 \mathrm{a}+4$ \\
& $\mathbf{1 0}$ & $\mathbf{1 2}$ & 14 & 16 & 18 & $\ldots$ & $2 \mathrm{a}+8$ \\
\hline
\end{tabular}

Table 2: The table for $n=3$

\begin{tabular}{l|llllllll}
\hline $\mathbf{x}$ & \multicolumn{1}{|c}{$\mathbf{y}$} & & & & \\
\hline 3 & 3 & 5 & 7 & 9 & 11 & 13 & $\ldots$ & \\
5 & 5 & 7 & 9 & 11 & 13 & 15 & $\ldots$ & \\
7 & 7 & 9 & 11 & 13 & 15 & 17 & $\ldots$ & \\
\hline & $\mathbf{x}$ & + & $\mathbf{y}$ & $=$ & $\mathbf{b}$ & & & \\
\hline & 6 & 8 & 10 & 12 & $\mathbf{1 4}$ & $\mathbf{1 6}$ & $\ldots$ & $2 \mathrm{a}+4$ \\
& 10 & 12 & $\mathbf{1 4}$ & $\mathbf{1 6}$ & 18 & 20 & $\ldots$ & $2 \mathrm{a}+8$ \\
& $\mathbf{1 4}$ & $\mathbf{1 6}$ & 18 & 20 & 22 & 24 & $\ldots$ & $2 \mathrm{a}+12$ \\
\hline
\end{tabular}

ements of $y$ groups that each group of $y$ contains $n$ pieces of consecutive odd numbers, the result will be like in the tables. Here, the first group of $y$ is the same with $x$ column. The first and the smallest number of the next $y$ group is greater by 2 than the first and the smallest number of the previous $y$ group. The sum of $b$ is always an even number as well.

As the first $y$ group is also $x$ group and as also the biggest number of $x$ group is always $2 n+1$, the biggest number of first $b$ group must always become $2(2 n+1)=4 n+2$. This number is the first number which starts to be common with all the other $b$ even numbers which are formed by different $x$ elements for the same $n$ value in the tables; because it is in the last emerging line. Each even number after this number can be formed absolutely as $n$ pieces the number itself is included as well. As 1 piece of selected consecutive $n$ pieces odd numbers must be prime number until $n+1$ group, minimum 1 piece of the even numbers which are in each even number group has $n$ pieces of the same even number must be sum of two prime numbers.

Here, if also $4 n+4$ number after $4 n+2$ number is included, if all of emerging $n$ pieces of the same $4 n+4$ even numbers are not the numbers of $n+1$ group in a table for the same $n$ value, as all results of $4 n+4$ and $4 n+2$ form set of even num- 
bers greater than 8 , then it means each even number which is greater than 8 absolutely must be sum of two prime numbers.

The equation between $a$ values that first $a$ namely $a_{1}$ which gives even numbers in the first line for $a=[1, \infty)$ over $2 a+4$, and $a_{n}$ which accepts the first even as $4 n+2$ and gives the numbers of $2 a+4 n$ is (1)

$$
a_{1}=a_{n}+2 n-2
$$

Here, result of $a_{1}$ for $a_{n}=1$ is also equal to number of used odd numbers in the first $y$ line to form $4 n+2$ number or is equal to number of used numbers which are different than each other in the tables for the same $n$ value, outside of using $a_{n}$ to form $2 a_{n}+4 n$ namely $4 n+2$ number; because it is also $x+y$ operation number of forming $4 n+2$ even number in the first line; therefore $a_{1}$ number over (1) for $a_{n}=2$ is required to form $4 n+4$, and it must be (2)

$$
N=2 n
$$

\subsection{The result}

As the result for the above stated information, assume that $4 n+2$ and $4 n+4$ numbers cannot be sum of two prime numbers for each $n$ value. Last $n$ pieces of $y$ consecutive odd numbers which are required to form $4 n+2$ and $4 n+4$ numbers for required $n$ value, must be $n$ group numbers; thus only $2 n-n=n$ pieces $y$ consecutive odd numbers can be used it means being 3 is the first usable number on the first $y$ line; but already this means that all numbers except 1 are non-prime consecutive odd numbers for each $n$ value that is impossible. Even if it is assumed that, for the worst possibility $n$ and $n-1$ groups emerge together by the same numbers as non-unique groups, also the information stated above that this assumption is impossible. Already if it is impossible even for $n$ group, when it is assumed that there are another groups,

$$
2 n-\sum_{n=2}^{n} n
$$

number of used or usable consecutive $y$ odd numbers will decrease by (3), and is impossible for $n>1$ definition. As and if we do not know prime separation, for the worst possibility of number of existent primes in $2 n$ pieces usable consecutive odd numbers on the first $y$ line, assume that for $n$, there are consecutive unique $n$ groups until last $2 n$ number even if actually they cannot be fitted such that as emerging number of the numbers will be bigger than $2 n$ by this way; but if it is right, then amount will not be important that you can assume that there are $(2 n)^{2}$ pieces $n$ groups if essence of the function provides this that it is provided here; because the number of the non-prime numbers will increase greater than the primes for assumed $n$ pieces unique groups; so as unique groups are between two primes, there must be $2 n$ pieces prime numbers. As this $2 n$ is also equal to number of the used numbers on the first $y$ line that it is not important which numbers of $2 n$ pieces numbers are prime or not prime here, absolutely minimum 1 piece of each $n$ pieces the same even $4 n+2$ numbers and minimum 1 piece of each $n$ pieces the same even $4 n+4$ numbers which emerge in each table separately are absolutely sum of two prime numbers. Also it means that all even numbers greater than 8 are sum of two prime numbers. This is also proof of infinite number of twin primes.

\section{Twin primes}

Select any unique number group which has $n$ pieces of consecutive non-prime odd numbers. This group has to exist between 2 prime numbers according to the definition stated at the beginning between prime numbers and group numbers; because otherwise there will occur a group like $n+1$ group instead of $n$ group that actually infinite number of non-prime numbers can be consecutive. For example, let us take consecutive multiples of 3,5, 7, 9 and 11 for $n=5$ group. Being $a$ is an odd number, any multiples of odd numbers become $a(2 x+1)$ for required $x$; so over $\left(6 x_{1}+3\right)+2=10 x_{2}+5$, it becomes $x_{1}=5 x$. Over $\left(6 x_{1}+3\right)+4=14 x_{3}+7$, it becomes $x_{1}=7 x$. Over $\left(6 x_{1}+3\right)+6=18 x_{4}+9$, it becomes $x_{1}=9 x$. Over $\left(6 x_{1}+3\right)+8=22 x_{5}+11$, it becomes $x_{1}=11 x$. Results of $6 x_{1}+3$ which are odd multiples of 3 become $30 x+3$, $42 x+3,54 x+3$ and $66 x+3$ for the stated $x_{1}$ values. If also these are made equal to each other, being $(11 \cdot 9 \cdot 7 \cdot 5 \cdot x)=m$ and the first number of the group is multiple of 3 , consecutive multiples of the group numbers become by order $6 m+3$, $6 m+5,6 m+7,6 m+9$ and $6 m+11$. For more consecutive odd multiples, we can increase the number of used numbers in a group forever.

I selected $n_{1}, n_{2}, n_{3}$ and $n_{4}$ consecutive odd numbers in $n=4$ group like $p_{1} n_{1} n_{2} n_{3} n_{4} p_{2}$ being $p$ is prime number. Minimum one of these $n$ numbers has to be multiple of 3 ; because separation of odd multiples of 3 is according to $6 x+3$, and so there are always 2 consecutive odd numbers between two consecutive odd multiples of 3 . Here, if $n_{2}$ becomes odd multiple of 3 , then $p_{2}$ must be the next multiple of 3 that this is only possible for $n=5$. If $n_{3}$ becomes odd multiple of 3 , then $p_{1}$ must be the previous multiple of 3 that this is also possible for $n=5$. As it was said, it is possible to form groups have infinite number of consecutive non-prime odd number, namely $n=4$ must exist anyway.

If $n_{1}$ becomes odd multiple of 3 , then $n_{4}$ must be the next multiple of 3 and also $n_{5}$ becomes the next multiple of 3 after $n_{4}$ as $n_{0}$ became the previous odd multiple of 3 before $n_{1}$ over $n_{0} n_{x} p_{1} n_{1} n_{2} n_{3} n_{4} p_{2} n_{y} n_{5}$.

If $n_{4}$ becomes odd multiple of 3 , then $n_{5}$ becomes the next multiple, and $n_{0}$ and $n_{1}$ become the previous multiples of 3; thus $n_{1}$ and $n_{4}$ are pretty suitable to be odd multiple of 3 .

Here, infinite number off odd consecutive $n$ number can take place after $n_{5}$; so element number of the next group after $n=4$ is not important; but $n_{y}$ is always prime or not, this is important. Over $n_{y}=n_{5}-2=(6 x+3)-2$, it becomes $n_{y}=6 x+1$. Hence, $n_{y}$ never can be only prime number where 
$x \in \mathbb{Z}^{+} \wedge x>0$. It is not prime for required $x$, and otherwise it is prime for emerging odd numbers between two $n_{y}$ and $n_{y+1}$ numbers which are a result of consecutive two $x$ and $x+1$ values; so when it becomes $n_{y}=p_{3}$, it is a twin prime group between $n_{4}$ and $n_{5}$; thus twin primes are in infinite number.

\section{Collatz Problem}

The main question about the Collatz Problem is also pretty clear. When a positive whole number is selected, if the number is an even number then it is divided by 2 ; otherwise it is multiplied by 3 , and after that 1 is added to the result. When the same operation with required option of the problem due to the condition of to be odd or even number of the result is repeated for the last results, can each positive integer which is different than 0 and 1 be reduced into 1 ?

\subsection{The solution}

If the input number is an even number, and if it is not an even number as $2^{n}$ as well for definition of $n \in \mathbb{Z}^{+} \wedge n>0$; being $p n$ is process number, when the input number is divided by $p n$ times 2 or directly by $2^{p n}$, each positive even number absolutely turns into a positive odd number as they can be defined as $(2 x+1) \cdot 2^{n}$ for definition of $x, n \in \mathbb{Z}^{+} \wedge n>1 \wedge x>0$; thus we should only work over odd numbers.

$$
a_{n+1}=\frac{3 a_{n}+1}{2}
$$

Over (4), it must become $a_{n+1}=\{2,5,8,11, \ldots ., 3 x-1\}$ where $x \in \mathbb{Z}^{+} \wedge x>0$ for a limited interval. For the numbers which make $a_{n}$ an odd number, it becomes $a_{n+1}=$ $\{5,11,17, \ldots, 6 x-1\}$ for a limited interval and the same $x$ definition. Also it becomes $a_{n}=\{3,7,11,15, \ldots ., 4 x-1\}$ over $a_{n+1}$ odd numbers for the same conditions.

The below is a table over $a_{n}$ and $a_{n+1}$ numbers by order over (4) for a limited interval being $\mathrm{E}$ is even and $\mathrm{O}$ is odd.

Table 3: $a_{n}$ and $a_{n+1}$ numbers

\begin{tabular}{l|lllllll}
\hline$a_{n}$ & 3 & 7 & 11 & 15 & 19 & 23 & $\ldots$ \\
$a_{n+1}$ & 5 & 11 & 17 & 23 & 29 & 35 & $\ldots$ \\
$a_{n+2}$ & $\mathrm{E}$ & $\mathrm{O}$ & $\mathrm{E}$ & $\mathrm{O}$ & $\mathrm{E}$ & $\mathrm{O}$ & $\ldots$ \\
\hline
\end{tabular}

On the table of 3 , for $12 x-7$ numbers from $a_{n+1}$ numbers with the same $x$ definition, it becomes (5).

$$
18 x-10=\frac{3(12 x-7)+1}{2}
$$

The result of (5) is absolutely even number for each $x$. For $12 x-1$ numbers from $a_{n+1}$ numbers with the same $x$ definition, it becomes (6).

$$
18 x-1=\frac{3(12 x-1)+1}{2}
$$

The result of (6) is absolutely odd number for each $x$. Right this point, the question is this that for (7),

$$
a_{n+2}=\frac{3 a_{n+1}+1}{2}
$$

when $a_{n+2}$ becomes an even number and divided by $2^{p n}$, does emerging odd numbers as a result always emerge before $a_{n+1}$ in set of odd numbers due to number order or can it be bigger number than $a_{n+1}$ ?

As the answer, if the result of the operation of (7) becomes even, to realize of to be reduced of the result of $\frac{3 a_{n+1}+1}{2 \cdot 2^{p n}}$ operation into an odd number which is before $a_{n+1}$, the condition of (8) always has to be provided.

$$
1>\frac{\frac{3 a_{n+1}+1}{2 \cdot 2^{p n}}}{a_{n+1}}
$$

If (8) is edited then as (9),

$$
1>\frac{1}{2^{p n+1}}\left(3+\frac{1}{a_{n+1}}\right)
$$

the inequality of (9) always provides this for the definition of $p n>0 \wedge a_{n+1}>1 \wedge p n, a_{n+1} \in \mathbb{Z}^{+}$.

As a result, when $a_{n+2}$ is reduced into an odd number, the odd number is always before $a_{n+1}$ odd, and is smaller than it. It means that the odd number as a result of $a_{m}=\frac{3 a_{n+1}+1}{2 \cdot 2^{p n}}$ is always smaller than $a_{n+1}$ number. Even if $\frac{3 a_{m}+1}{2}$ becomes even number, again it can be reduced into a smaller odd number than both $a_{m}$ and $a_{n+1}$, and it is acceptable for the other repeats as well.

Right this point, a second question emerges that is there a number which always gets bigger and does not become an odd number on (4) infinite chain.

As the answer, there is table for the numbers which do not emerge on table 3 . If these numbers are included to the numbers on table 3 as well, the sum is set off odd numbers. There will no other odd number which is not included to the calculations.

Table 4: The numbers which are not in the previous table

\begin{tabular}{lllllllll}
\hline $\mathbf{5}$ & 9 & 13 & $\mathbf{1 7}$ & 21 & 25 & $\mathbf{2 9}$ & $\ldots$ & $4 \mathrm{x}+1$ \\
\hline
\end{tabular}

The numbers which are written thick are also in $a_{n+1}$ line in table 3. The other numbers are the numbers which are not in table 3.

If some groups are made for $4 x+1$ numbers in table 4 , there will only emerge 3 groups for $12 x-3,2 x+1$ and $12 x-7$ numbers for the same $x$ definition. Being $a_{n+1}=12 x-3$, (10) always gives even result. 


$$
18 x-8=\frac{3(12 x-3)+1}{2}
$$

Being $a_{n+1}=12 x+1$, (11) always gives even number result as well.

$$
18 x+2=\frac{3(12 x+1)+1}{2}
$$

As $12 x-7$ numbers, they are already the same numbers with $a_{n+1}$, and at the result of (7) they always give even number being $a_{n+1}=12 x-7$.

As even numbers, they can always be reduced into a smaller odd number than the odd number which makes them even number in the operation of (4) as it was proved; thus if each one of $a_{n}=4 x-1$ numbers do not get greater by turning into an odd number when (4) is repeated for each $n$ number where $n \in \mathbb{Z}^{+}$, it means all positive whole numbers different than 0 and 1 can be reduced into 1 .

In table $3,8 x-5$ numbers from $a_{n}$ numbers turn into $a_{n+1}$ number which gives even result over (7); thus the only chance is to give odd result always of $8 x-1$ numbers over (4) infinite chain. To be realized of this, the same numbers with $a_{n}$ must emerge on $a_{n+1}$ line in table 3. Also always the numbers which give odd result must emerge on $a_{n+1}$ line between them. For the same $x$ condition, $4 x-1$ and $6 x-1$ operations give $a_{n}$ and $a_{n+1}$ over (4) for the same $x$ number; so the waited loop occurs or does not occur,

$$
x_{1}=\frac{6 x_{2}}{4}
$$

(12) shows this over the equality of $4 x_{1}-1=6 x_{2}-1$. For (12), it becomes $x_{1}=3 t$ and $x_{2}=2 t$ over $t \in \mathbb{Z}^{+} \wedge t>0$ condition; so the problem is reduced into the rule of table 5 below.

Table 5: The numbers for each $\mathrm{t}$

\begin{tabular}{l|lllllll}
\hline $\mathbf{2 t}$ & 2 & 4 & 6 & 8 & 10 & 12 & $\ldots$ \\
$\mathbf{3 t}$ & 3 & 6 & 9 & 12 & 15 & 18 & $\ldots$ \\
\hline
\end{tabular}

Each number on $4 x-1$ and $6 x-1$ is also order number of $a_{n}$ and $a_{n+1}$ in table 5; thus the number has $3 t$ order number on $a_{n}$ or $2 t$ line and the number has $2 t$ order number on $a_{n+1}$ or $3 t$ line in table 5 are the same numbers.

As the odd numbers on $3 t$ line in table 5 are $a_{n+1}$ numbers which give even result in table 3 , they are elected; thus table 5 turns into table 6 .

Table 6: The other numbers for each $t$

\begin{tabular}{l|lllllll}
\hline $\mathbf{4 t}$ & 4 & 8 & 12 & 16 & 20 & 24 & $\ldots$ \\
$\mathbf{6 t}$ & 6 & 12 & 18 & 24 & 130 & 36 & $\ldots$ \\
\hline
\end{tabular}

In table 6 , to occur of the infinite loop, when a $4 t$ number is selected, also $6 t$ number which is under it and on $6 t$ line in table 6 must be even number, and also this $6 t$ number must take place on $4 t$ line again. This condition has to take place for one or more than one number to be broken of the Collatz's reducing chain, and then one or more than one number will not be reduced into 1; but this is impossible; because for $t_{n+1}=\frac{6 t_{n}}{4}$ where $t>0 \wedge t, n \in \mathbb{Z}^{+}$, for each $t$ whole number,

$$
t_{n+1, t}=\lim _{n \longrightarrow \infty} \frac{6 t_{n, t}}{4}
$$

(13) has to be provided for the condition of (14),

$$
t_{n, t}, t_{n+1, t} \in \mathbb{Z}^{+}
$$

where $t_{n, t}=4 t, t$ is the order number of the number which is waited of starting the loop from it, and $n$ is the repeat number of (13) for each $t$. As this condition of (14), it cannot be provided for each $t$. For example, for $t_{1,1}=4$, it becomes $6 t_{1,1}=4 t_{2,1}$ and so becomes $t_{2,1}=6$. For $t_{2,1}=6$, it becomes $6 t_{2,1}=4 t_{3,1}$ and so becomes $t_{3,1}=9$. For $t_{3,1}=9$, it becomes $6 t_{3,1}=4 t_{4,1}$ and so becomes $t_{4,1}=27 / 2$. As it can be seen, $t_{4,1} \notin \mathbb{Z}^{+}$and so the condition of (14) cannot be provided.

\subsection{The result}

(13) cannot continue forever; because wee need a number which has infinite number of common divisors like $4^{\infty}$ or $(2 x+1) \cdot 4^{\infty}$ imaginary numbers. As it can be seen, only we can increase the repeat number by using a $t$ number like $4^{m}$ where $m>0 \wedge m \in \mathbb{Z}^{+}$that if $m$ gets bigger, then the repeat will increase; but there is no infinite repeat; hence, any whole number absolutely can be reduced into 1 by changing operation numbers of the Collatz's rule due to the used number.

For the repeats of (4), being $2^{m}$ is order number of the selected odd number of $a_{n}$ in table 3 where $m>0 \wedge m \in \mathbb{Z}^{+}$, it gives $m+2$ pieces odd number, and then the last one gives even number on (4) for $2^{m}$. Table 7 is a demonstration of this.

Table 7: The other numbers for each $t$

\begin{tabular}{l|lllll}
\hline $\mathbf{m}$ & & & & $\mathbf{a}_{\mathbf{n}}$ & \\
\hline $\mathbf{1}$ & $7 \longrightarrow$ & $11 \longrightarrow$ & 17 & & \\
$\mathbf{2}$ & $15 \longrightarrow$ & $23 \longrightarrow$ & $35 \longrightarrow$ & 53 & \\
$\mathbf{3}$ & $31 \longrightarrow$ & $47 \longrightarrow$ & $71 \longrightarrow$ & $107 \longrightarrow$ & 161 \\
$\cdot$ & & & & & \\
$\cdot$ & & & & & \\
\hline
\end{tabular}

Also we can use an operation like (15), and we can derive another operation as well. The below is an example.

$$
a=7+\sum_{m=3}^{m} 2^{m}
$$

Here for (15), $m+1$ pieces odd numbers or repeats on (4) emerges being $a=a_{0}$ which is first input number on (4). You can write your own operation as well. 


\section{References}

1. Kavak M. 2017. Complement Inferences on Theoretical Physics and Mathematics. Open Science Framework. Available online: osf.io/tw52w

2. Kavak M. 2016. On the Uncertainty Principle. American Journal of Physics and Applications. Vol. 4, No. 4, pp. 90-123 